\title{
Prototype Design of Speed Detection Mobile Application for Golfers Swing Movement using Computer Vision Compared to Portable Radar and Accelerometer Systems
}

\author{
Bagus Wibowo \\ Edi Sofyan \\ Gembong Baskoro \\ Department of Mechanical Engineering Department of Mechanical Engineering Department of Mechanical Engineering \\ Swiss German University \\ Swiss German University \\ Tangerang 15143, Indonesia \\ Tangerang 15143, Indonesia \\ Swiss German University \\ Tangerang 15143, Indonesia \\ Email: wibowb@gmail.com
}

\begin{abstract}
Prototype Design of Golf Swing Speed Detection Mobile Application (GSSDMA)/Swing Vision (SV) has been researched and developed with computer vision technique. Frames detection method has been implemented to performed calculation of the swing speed by manually identification of the frames from start of down swing to impact of the ball with Matlab Video Viewer as initial reference calculation, when head of golf club start to move to downswing as framezero/fro and frame-n/frn as end of the frame after the head of golf club impact to the ball and then the total frames can be determined by frn minus fro (frn - fro) which will be used for speed calculation reference formula using Python programming. Both measurements have been recorded using RADAR and accelerometer systems to get references of swing speed data measurement from some golfers in golf driving ranges. Accelerometer data measurements have been selected to use as reference of speed calculation with Python programming for software application development since deviation standard is lower than the RADAR systems. There is a limitation on the hand phone camera speed which only have thirty frames per second ( $30 \mathrm{fps}$ ) and the maximum swing speed can be tested with this camera is $101,2 \mathrm{mph}$ at the moment which has three frames (frn-fro). Found a swing speed formula $y=-0,53 x^{3}+9,53 x^{2}-61,27 x+213,35$ from experimental datas of Driver, 6 Iron, 8 Iron and Pitching and maximum swing speed can be predicted is $124,69 \mathrm{mph}$ which has two frames.
\end{abstract}

Keywords: Computer Vision, Swing Speed Detection, Frames Detection, RADAR, Accelerometer.

\section{INTRODUCTION}

Computer vision technology is part of Artificial Intelligent/AI has been growing considerably during the past decade and has been used a significant role in scientific, industrial, sport, space and government applications. Previous technology as such as accelerometer sensor and RADAR systems has been growing very importance for speeds measurement and both systems have been used until now for speed monitoring of vehicles on high ways, ships on oceans, engines monitoring, sport competitions, aero planes, etc.

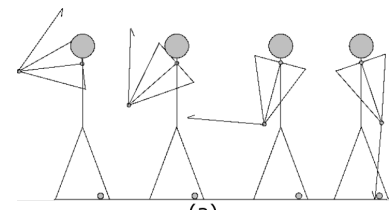

(a)

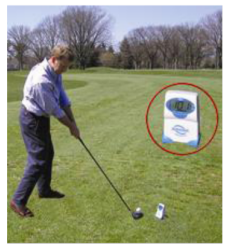

(b)

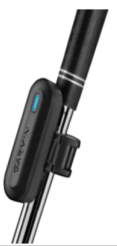

(c)
Fig. 1. (a) Golf Swing, (b) Swing Speed Radar, (c) Garmin Accelerometer.

\section{A. Background}

Motivation of this research will design a portable speed detection system using hand phone camera with computer vision technique for golf swing movement (Fig.1a.) as an alternative technology with more simple equipment and be more cost effective compare to the both systems. Mobile application has been very popular and useful in last decades especially using hand phone and very good for start-up business. If the speed measurement using hand phone camera with computer vision is accurate enough and more practicable, so will be designed an application on hand phone camera with computer vision technique.

\section{B. Objectives}

Golf Swing Speed Radar (Fig.1b.) is a sensor using microwave Doppler radar velocity that measures the swing speed 
of golfers. The Golf swing RADARs measurement is still debatable if compare to accelerometer. Garmin TruSwing (Fig.1c.) is an accelerometer sensor which attach to the shaft of club just below the grip and need to do data transfer to hand phone via blue tooth communication media and also need an internet connection to know the old swing datas.

\section{Significance of Study}

A speed detection system using hand phone camera with frames detection method on swing of golf sport is more simple and effective systems compared to both systems RADAR and accelerometer.

\section{Research Questions}

Question 1: How the standard mobile phone with $30 \mathrm{fps}$ of camera can detect the swing speed of golfer?

Question 2: How the swing speed of golf player can be calculated?

\section{LITERATURE REVIEW}

\section{A. Theoretical Perspectives}

On this thesis will collect some video datas from experimental of swing golfers become a data base references, and these datas will be used to predict the swing speed with 3rd degree polynomial linear regression formula as follow:

$$
y=p_{1} x^{3}+p_{2} x^{2}+p_{3} x+p_{4}
$$

where: $y=$ Swing Speed, $x=$ Total Frames, $p_{1}, p_{2}, p_{3}$ and $p_{4}$ are coefficients $3^{\text {rd }}$ degree polynomial.

A standard deviation formula will be used to compare measurements among RADAR, Accelerometer and (GSSDMA)/Swing Vision (SV). In digital imaging, a pixel or picture element is a physical point in a raster image, or the smallest addressable element in an all points addressable display device; so it is the smallest controllable element of a picture represented on the screen. Pixels, abbreviated as "px", are also a unit of measurement commonly used in graphic and web design, equivalent to roughly 196 inch $(0.26 \mathrm{~mm})$. Computer Vision is a knowledge and technology of computer systems which has a capability to perform acquisition, processing, understanding from an image or streaming video, so the following are simple explanation of the Computer Vision.

ComputerVision $=$ Camera + Computer + PatternRecognition

And a technology called Machine Vision has capability for measurement, counting, and decoding and on this thesis is using frames detection method to do speed calculation. Frame rate (expressed in frames per second or fps) is the frequency (rate) at which consecutive images called frames appear on a display. The term applies equally to film and video cameras, computer graphics, and motion capture systems. The standard mobile phone video camera is $30 \mathrm{fps}$ or 1 frame need 0.03 milliseconds.

\section{B. Previous Studies}

1) Speed Detection Camera System using Image Processing Techniques on Video Streams: This paper, presents a new Speed Detection Camera System (SDCS) that is applicable as a radar alternative. SDCS uses several image processing techniques on video stream in online -captured from single cameraor offline mode, which makes SDCS capable of calculating the speed of moving objects avoiding the traditional radars' problems. SDCS processes can be divided into four successive phases; first phase is Objects detection phase. The second phase is Objects tracking, which consists of three successive operations, Object segmentation, Object labelling, and Object canter extraction. Third phase is speed calculation phase, which is calculated from the number of frames consumed by the object to pass-by the scene. The final phase is Capturing Object's Picture phase, which captures the image of objects that violate the speed limits.

2) Synthesis of a stroboscopic image from a hand-held camera sequence for a sports analysis: This paper presents a method for synthesizing a stroboscopic image of a moving sports player from a hand-held camera sequence. This method has three steps: synthesis of background image, synthesis of stroboscopic image, and removal of players shadow. In synthesis of background image step, all input frames masked a bounding box of the player are stitched together to generate a background image. In synthesis of stroboscopic image step, the background image, the input frame, and a mask of the player synthesize a stroboscopic image. In removal of shadow step, we remove the players shadow which negatively affects an analysis by using mean-shift. In our previous work, synthesis of background image has been time consuming. In this paper, by using the bounding box of the player and by subtracting the images for synthesizing a mask, computational speed and accuracy can be improved. In experiments, we confirmed the electiveness of the proposed method, measured the players speed and stride length, and made a footprint image.

\section{RESEARCH Methods}

Frames detection method has been selected to performed calculation of the swing speed by manually identification of the frames from start of down swing to impact of the ball with Matlab Video Viewer as initial reference calculation, when head of golf club start to move to downswing as frame zero/fr0 at just above of the golfer head and frame $n / f r n$ as end of the frame after the head of golf club impact to the ball and then the total frames can be determined by fn minus fro (frn - fro) which will be used for speed calculation reference formula using Python programming.

\section{A. Materials and Equipment}

MATLAB R2018a and Video Viewer will be used to perform frames identification, frame source rate can be check on Matlab Video Viewer as Figure 2 below, for example source frame rate is 29.9833 frames/sec. 


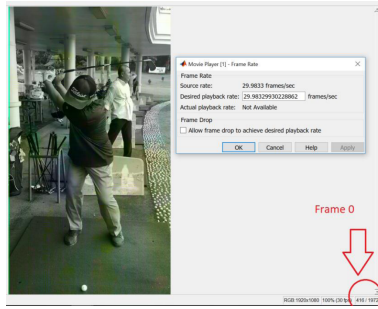

Fig. 2. MatLAB Video Viewer.

Software Programming and libraries: Python 3.7.3, Flask 1.0.2, Numpy 1.16.3, Matplotlib 3.0.3, Pandas 0.24.2, Opencvpython 4.1.0.25, Imutils 0.5.2, Opencv-Contrib-python 3.3.0.9. Hand phones: Samsung Galaxy J6+ Model SM-J610F/DS with Android version 8.1.0. and iPhone 6 64410.

Speed measurement tool 1: Garmin TruSwing Golf Swing Analyzer with Garmin Connect application software on hand phone and will use Bluetooth media communication for data transfer from sensor to the hand phone.

Speed measurement tool 2: Swing Speed RADAR Model No. SSR364

\section{B. Speed Calculation}

The objects speeds can be calculated by detecting the first frame which the object has entered the scene / start down swing at (Frame-0) and keeping track of the object till it leaves the scene /before impact to the ball at frame (Frame-n).

\section{Data Collection and Formula}

The formula of how many miles/kilometer per frame refers to data collections from swing measurements. Data Collection will be done with full swing of pitching, 8 iron, 6 iron and driver.

\section{Performance Test}

- RADAR and accelerometer will be used to prove the swing speed.

- Troubleshooting for system inconsistencies.

- Function testing.

\section{RESUlT AND Discussions}

Some experiments of golf swing measurements by some of golf players have been done at golf driving ranges with using Driver, 6 Iron, 8 Iron and Pitching (Fig.3) to get some datas as reference with using both RADAR and Accelerometer measurements. The experimental only be done with full swing not included half swing since the swing speed measurement usually will be used to know the maximum of a ball distance from the ball take off /impact until the ball landed on the address and then will be used to choose the type of golf club shaft to get the best optimum performance of the golf player

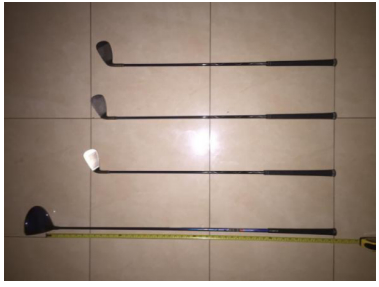

Fig. 3. Driver, 6 Iron, 8 Iron and Pitching Clubs.

\section{A. Initial Evaluation}

Frames detection method will be implemented since 30 fps of camera speed is already factory calibrated. Another method is pixel calculation and will not applicable for mobile application which need a fix distance between camera and golfer and then will be based on pixel counting calculation and one pixel equivalent to roughly 196 inch $(0.26 \mathrm{~mm})$.

\section{B. Data Analysis}

The following are some data experiments of swing speed measurements using some golf club Pitching, 8-Iron, 6-Iron and Drivers. Refer to experimental datas the accelerometer will be used as reference since repeatability and deviation standard better than RADAR and then why RADAR measurement result out of the maximum range on Figure 4. The results are radar measurement $146.3 \mathrm{mph}$ (avg.), radar deviation standard: 8.166, accelerometer measurement $101.2 \mathrm{mph}$ (avg.), accelerometer deviation standard: 1.135 , and average frames counted manually 3 frames.

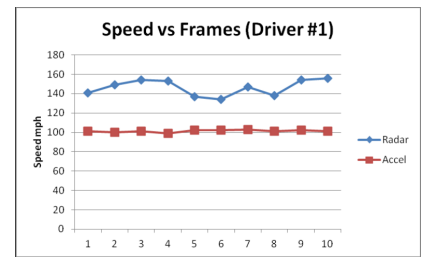

Fig. 4. Graphic swing speed measurementwith Driver 1.

The standard mobile phone video camera is $30 \mathrm{fps}$ or 1 frame need a 0.03 milliseconds. Maximum swing speed can be achieved is $124,69 \mathrm{mph}$ which has two frames from formula $y=-0,53 x^{3}+9,53 x^{2}-61,27 x+213,35$ which $x$ is frames counted and $y$ is a swing speed.

\section{Function Testing}

The following are function testing the python program of swing speed measurement with frame detection method using some golf clubs: Pitching, 8-Iron, 6-Iron and Driver 2 and Driver 1 . 
TABLE I

ACCELEROMETER VS SWING VISION

\begin{tabular}{|c|c|c|c|c|c|}
\hline $\begin{array}{l}\text { Club } \\
\text { Name }\end{array}$ & Manua & $\begin{array}{l}\text { Detection } \\
\text { Speeds } \\
\text { Accel } \\
\text { (mph) }\end{array}$ & Frames & $\begin{array}{l}\text { DMA } \\
\text { Speeds } \\
\text { Swing } \\
\text { Vision } \\
\text { (mph) }\end{array}$ & Deviation \\
\hline Driver \#1 & 3 & 101 & 3.046 & 100.093 & -0.89802 \\
\hline Driver \#2 & 4 & 86,5 & 4.031 & 86.360 & $-0,16185$ \\
\hline 6 Iron & 5 & 78 & 5.031 & 78.514 & 0,658974 \\
\hline 8 Iron & 6 & 73,6 & 6.031 & 73.638 & 0,05163 \\
\hline PITCHING & 7 & 68,8 & 7.046 & 68.432 & $-0,5348$ \\
\hline
\end{tabular}

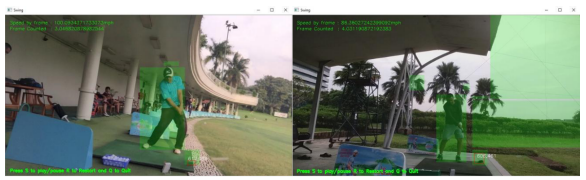

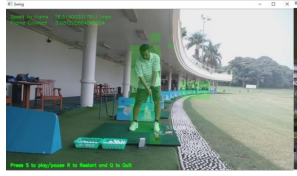

(c)

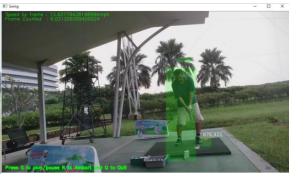

Fig. 5. Function Testing of Swing Speed of (a) Driver 1 Frames 100,093 mph with Frames Counted 3,046. (b) Driver 2 by Frames $86.360 \mathrm{mph}$ with Frames Counted 4.031. (c) Iron 6 by Frames $78.514 \mathrm{mph}$ with Frames Counted 5.031 (d) Iron 8 by Frames $73.638 \mathrm{mph}$ with Frames Counted 6.031 .

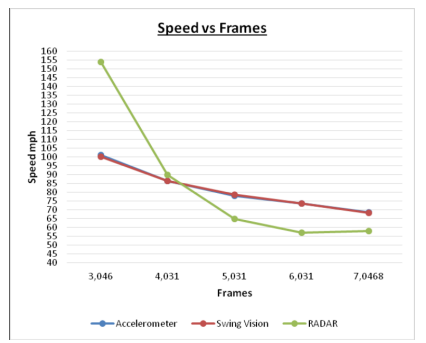

Fig. 6. Speed Vs Frames by RADAR, Accelerometer and Swing Vision.

\section{CONCLUSIONS AND RECOMMENDATIONS}

Prototype Design of Golf Swing Speed Detection Mobile Application (GSSDMA)/Swing Vision (SV) has been researched and developed with computer vision technique. Accelerometer measurement datas have been selected to use as reference of speed calculation with Python programming for software application development since deviation standard is lower than the RADAR systems.
TABLE II

Strengths, Weaknesses and Interesting of Swing Vision

\begin{tabular}{llll}
\hline No. & Strengths & Weaknesses & Interesting \\
\hline 1 & $\begin{array}{l}\text { Simple } \\
\text { Equipment } \\
\text { without } \\
\text { additional } \\
\text { hardware }\end{array}$ & $\begin{array}{l}\text { Low speed camera } \\
30 \mathrm{fps}\end{array}$ & $\begin{array}{l}\text { Using CV Algorithm } \\
\text { with Machine Vision } \\
\text { Technology }\end{array}$ \\
\hline 2 & $\begin{array}{l}\text { High Accuracy } \\
\text { depend on master } \\
\text { calibrator }\end{array}$ & $\begin{array}{l}\text { Maximum speed } \\
124,69 \mathrm{mph} \text { only }\end{array}$ & $\begin{array}{l}\text { Using Video can be } \\
\text { used to know how the } \\
\text { swing movement }\end{array}$ \\
\hline 3 & $\begin{array}{l}\text { Cheap using } \\
\text { Python free } \\
\text { programming } \\
\text { software }\end{array}$ & $\begin{array}{l}\text { Calibration based new data of } \\
\text { on neasurements }\end{array}$ & $\begin{array}{l}\text { Can be developed to } \\
\text { correct wrong move- } \\
\text { ment of the golfer }\end{array}$ \\
\hline
\end{tabular}

\section{A. Conclusions}

The GSSDMA/SV with hand phone camera can be used as an alternative speed measurement of the golf swing movement but there is a limitation on the hand phone camera speed which only have thirty frames per second (30 fps) as standard specification nowadays. The maximum speed swing speed has been be tested is $101,2 \mathrm{mph}$ at the moment which has three frames (frn-fr0). Found a swing speed formula $y=-0,53 \times 3$ $+9,53 \times 261,27 x+213,35$ from data experimental of Driver, 6 Iron, 8 Iron and Pitching. Maximum swing speed can be predicted is $124,69 \mathrm{mph}$ which has two frames.

\section{B. Recommendations}

Continuing the development of GSSDMA/SV is highly recommended especially if the mobile frame rate has increased with minimum $75 \mathrm{fps}$, since it will increase accuracy. In order to get more accurate measurements, combination of frames detection method and various image processing methods can be researched and developed. Why RADAR measurement result out of the maximum range on figure 11 ? this question need to do another research in regard to RADAR measurement, but on figure 10 the result almost similar between RADAR and accelerometer.

\section{ACKNOWLEDGMENTS}

I would like to thank to Mr. Edi Sofyan, B.Eng, M.Eng, Ph.d and Mr. Dr. Ir. Gembong Baskoro, M.Sc, my research guides, for giving me opportunity to work on this research. Under their guidance I was able to complete this research in a short period of time and also gave me various ideas for making this research successful.

\section{REFERENCES}

[1] Osman Ibrahim, Hazem ElGendy, and Ahmed M. ElShafee, Speed Detection Camera System using Image Processing Techniques on Video Streams. International Journal of Computer and Electrical Engineering, Vol. 3, No. 6., December 2011.

[2] Kunihiro Hasegawa, Hideo Saito, Synthesis of a stroboscopic image from a hand-held camera sequence for a sports analysis. Keio University, Yokohama, 223-8522, Japan. Computational Visual Media DOI 10.1007/s41095-016-0053-5 Vol. 2, No. 3, 277289. September 2016

[3] Zhiwei LI, Study on Dynamic Analysis and Wearable Sensor System for Golf Swing. A Thesis Submitted for the Degree of Doctor of Philosophy. Kochi University of Technology, Present on Spring 2015. 